$\begin{array}{ll}\text { le portiQue } & \text { Le Portique } \\ \text { Revue de philosophie et de sciences humaines }\end{array}$

$30 \mid 2013$

Écrire sur l'art

\title{
Vers une pensée biblique
}

Jean-Paul Resweber

\section{OpenEdition}

Journals

Édition électronique

URL : http://journals.openedition.org/leportique/2661

DOI : $10.4000 /$ leportique.2661

ISSN : $1777-5280$

\section{Éditeur}

Association "Les Amis du Portique"

Édition imprimée

Date de publication : 18 juillet 2013

ISSN : 1283-8594

\section{Référence électronique}

Jean-Paul Resweber, « Vers une pensée biblique », Le Portique [En ligne], 30 | 2013, document 5, mis en ligne le 11 octobre 2013, consulté le 26 mars 2021. URL : http://journals.openedition.org/leportique/ 2661 ; DOI : https://doi.org/10.4000/leportique.2661

Ce document a été généré automatiquement le 26 mars 2021.

Tous droits réservés 


\title{
Vers une pensée biblique
}

\author{
Jean-Paul Resweber
}

\section{RÉFÉRENCE}

Nice, Ovadia, 2020, 244 pages

Nice, Ovadia, 2012, 290 pages.

1 Bernard Grasset est l'auteur de plusieurs recueils de poésie, d'une traduction et de trois essais philosophique qui mettent en œuvre une pensée méditative et poétique, qui se situe à la croisée de deux chemins : celui de la pensée biblique et celui de la pensée philosophique, ou, comme aime à le souligner l'auteur, celui de l'exégèse biblique et celui de la philosophie. Bernard Grasset inaugure sa recherche dans un essai intitulé "Les pensées de Pascal, une interprétation de l'Écriture » (Kimé, 2003), puis dans un autre essai qui prolonge ce dernier : "Vers une pensée biblique » (Ovadia, 2010), enfin dans un essai intitulé «Bible, Sagesse et Philosophie» (2012) qui reprend, en les approfondissant, les deux premiers. Ces trois livres constituent les volets d'un triptyque dont la réalisation est commandée par la même source d'inspiration philosophique et religieuse.

2 Dans son livre Vers une pensée biblique, Bernard Grasset se propose d'illustrer ce qu'il entend par la pensée biblique. Il s'emploie à extraire les «traces bibliques » présentes chez des philosophes, familiers de la lecture biblique, comme le sont les Pères de l'Église, notamment Grégoire de Nazianze et, plus proche de nous, Pascal, puis chez des philosophes, qui, comme L. Lavelle et G. Marcel ne sont pas des « biblistes ». Il mobilise un double geste pour s'acquitter de cette tâche. Le premier s'exerce à l'endroit d'une pensée philosophique qui se déploie à partir et à l'intérieur la Bible : ainsi la philosophie antique se présente-t-elle comme une propédeutique intellectuelle à la "paideia " religieuse, puisque la religion est la vraie philosophie (philosophia vera religio est), mais aussi comme une initiation morale à la pensée biblique, puisque le travail de la pensée exige une ascèse qui implique l'attitude de l'eusébeia, cette piété de la pensée «qui éloigne de tout ce qui est artificiel » et qui est « un sentiment profond où se conjuguent 
ferveur religieuse, orthodoxie de la pensée, droiture morale et fidélité à la Parole " (p. 31). Pascal auquel l'auteur consacre de très belles pages (chapitre $2:$ Une esthétique pascalienne) opposant l'artifice à l'art du cœur se présente comme étant le parangon de cette démarche, selon laquelle le message biblique est non seulement le principe, mais aussi la fin de la pensée. Mais ce geste d'inspiration plutôt « archéologique » n'exclut pas un autre geste à portée plutôt "eschatologique", qui, sans pour autant se substituer au premier, exige de penser avec et au-delà de la Bible, en laissant à la pensée la liberté d'en approfondir les données et de construire des concepts de référence qui déplacent et dépassent, en les subsumant, les notions religieuses, à l'instar de L. Lavelle et de G. Marcel, le premier nous proposant un humanisme fondé sur une pensée de l'amour et le second un humanisme fondé sur la méditation du mystère.

3 Mais la réflexion de B. Grasset ne se limite pas à conjuguer ces deux axes. Assurant l'interface ou l'intersection entre la philosophie et la Bible, elle dessine un « milieu » de rencontre constitué par une référence dominante et déterminante : la quête passionnée de la sagesse. C'est dans ce milieu sapientiel, carrefour d'expériences intellectuelles et spirituelles, que peut se déployer l'aventure d'une pensée symbolique qui «lit poétiquement la réalité » (p. 231). Il s'agit finalement d'ouvrir un chemin de pensée qui découvre à l'homme la transcendance qui l'habite secrètement.

Poursuivant son dialogue entre culture hellénique et culture judéo-chrétienne, dans son livre Bible, sagesse et philosophie, où il mobilise en contrepoint exégèse et philosophie, B. Grasset entreprend une interprétation rigoureuse de la notion cardinale de sagesse, qui est au principe et à la fin de l'expérience religieuse et philosophique. Dans une première partie de son ouvrage, il analyse méticuleusement le modèle de la sagesse à partir des livres de la Bible hébraïque (hokhmah) et de son interprétation (sophia) dans la traduction des LXX. C'est à faire l'expérience pédagogique de la Sagesse que nous convie B. Grasset, car la Sagesse est à la fois enseignante et enseignée. Les grandes étapes de cette quête étrangement familière surgissent à des carrefours, où l'initié se trouve confronté à la Loi, à la Vérité, au cœur de la Présence (Schekhinah) de l'Être, et finalement à la Parole (Logos/Dabar).

5 Ainsi, la Sagesse est-elle le lieu de rencontre de l'expérience spirituelle et intellectuelle, de la pensée biblique et de la pensée philosophique : elle est la source d'un étonnement "thaumazein" qui fait battre le cœur du désir profond de l'homme, qui le rend insatiable d'une vérité qui est quête indéfinie (darash/zêtein), d'une vérité qui parle aussi bien à l'intelligence qu'à la foi et qui mobilise le même amour sapientiel (philein sophian). Mais il n'y a pas pour autant une double vérité scientifique et théologique, humaine et divine, comme le prétendait au XIII siècle le théologien Siger de Brabant. Bernard Grasset nous montre pourquoi et comment ces deux formes de sagesse, en se croisant, constituent l'architrave axiologique de notre culture occidentale. Que l'on emprunte la voie de la patristique, celle de saint Ephrem, de Clément d'Alexandrie, de Bernard de Clairvaux, de saint Bonaventure, de saint Thomas ou la voie des philosophes, comme celle qu'ont suivie Pascal, L. Lavelle ou G. Marcel, force nous est de constater que l'expérience philosophique de la Sagesse s'accomplit dans l'expérience religieuse d'une Sagesse qui est Dieu même. Si la vérité philosophique est dévoilement (alêtheia) et la vérité biblique fidélité (émet), on reconnaîtra que la seconde est au-delà de la première et que le dévoilement de l'alêtheia se transforme en écoute de la parole du Dieu fidèle. Mais on se tromperait à opposer pour autant ces deux voies et à penser que cette écoute se réaliserait dans la possession d'une vérité qui échapperait au jeu 
indéfini du voilement et du dévoilement. Le chemin de la Sagesse est celui de l'exode qui s'offre à l'homme soumis à l'épreuve de l'exil (p. 40).

6 Ce livre comporte une problématique qui réinterprète, en s'appuyant sur l'exégèse biblique, les rapports entre la philosophie et la théologie. L'exégèse biblique, en effet, assure la médiation entre ces deux disciplines : assurant le lien entre la philosophie et la théologie biblique, elle se transmue en une "philexégèse ", qui donne une profondeur insoupçonnée à l'interprétation de la Bible et, du même coup, offre à la philosophie un lieu de ressourcement de la pensée. Il s'agit certes d'une exégèse, car cet échange des idiomes se réalise au plan de la langue : la langue philosophique et la langue biblique s'enrichissent au contact l'une de l'autre. Mais cette exégèse qui se coule dans la logique du déchiffrement pascalien est en fait " une herméneutique du cœur ». Chaque langue organise son tracé à partir des traces qui résultent des emprunts qu'elle a faits à l'autre langue. Le lecteur trouvera une illustration paradigmatique de ce parcours en se reportant aux pages, où l'auteur se livre à l'exégèse du célèbre texte de l'Exode $(3,14)$ : "Je serai qui je serai » (('éhyéh 'asher 'éhyéh / Egô eimi ho ôn).

7 Ce qui, au final, constitue le motif de cette recherche, c'est sans doute le projet de présenter une anthropologie d'inspiration sapientielle résultant de l'entrelacs d'une anthropologie biblique et d'une anthropologie philosophique : « La philosophie biblique déchiffre l'humain dans l'éclaircie du divin. Au centre de son anthropologie figureront le cœur et l'esprit » (p. 84). Mais le livre de B. Grasset n'est pas seulement une réflexion intellectuelle, il est aussi un témoignage : le sujet de l'énonciation, à peine dissimulé dans le sujet scripteur, nous entraîne avec lui dans la recherche de la sagesse de l'amour et du Logos: "Comprendre le divin et l'humain comme amour, esprit et mystère, tisse la voie sapientielle... Comme le poète, le philosophe peut devenir un témoin spirituel du mystère... La pensée biblique advient comme profondeur de la philosophie » (p. 262).

8 Bernard Grasset ouvre un chantier de recherche intéressant et stimulant qui touche aux fondements mêmes de notre culture occidentale. Certes, les traces bibliques sont nombreuses chez ce qu'il appelle les philosophes non biblistes: Kant, connu pour ses annotations bibliques, pourrait figurer avec Kierkegaard, aux côtés de L. Lavelle et de G. Marcel. Chez tous ces philosophes, les traces s'organisent en un tracé, tissent une trajectoire et l'on peut suivre leur chemin de pensée, pour ainsi dire, à la trace. On ne s'étonnera pas pourtant de découvrir aussi des traces bibliques, cette fois cachées parce que recouvertes, chez des philosophes athées comme Spinoza, Hegel, Sartre et Heidegger. Mais on n'hésitera pas à élargir le champ d'investigation et à appliquer un traitement analogue à d'autres disciplines: ce qui nous conduirait à découvrir des traces bibliques serties dans les notions fondamentales du droit, de la morale et jusque dans le lexique de la langue. Tout se passe comme si ces diverses traces servaient de matrice à l'éclosion de concepts ou de philosophèmes qui, en les surdéterminant, présidaient du même coup à leur effacement. C'est à un vaste et passionnant travail de déchiffrage que nous invite Bernard Grasset. 